\title{
Biomarker Testing: Piercing the Fog of Alzheimer's and Related Dementia
}

\author{
Denis Horgan $^{a}$ Flavio Nobilib, c Charlotte Teunissen ${ }^{d}$ Timo Grimmer ${ }^{\mathrm{e}}$ \\ Dinko Mitrecic $^{f} \quad$ Laurence Ris $^{g} \quad$ Zvezdan Pirtosek $^{h} \quad$ Chiara Bernini $^{a}$ \\ Antonio Federico ${ }^{i}$ Daniel Blackburn ${ }^{j}$ Giancarlo Logroscinok \\ Nikos Scarmeas ${ }^{\prime}$ \\ aEuropean Alliance for Personalised Medicine, Brussels, Belgium; ${ }^{b}$ Department of \\ Neuroscience (DINOGMI), University of Genoa, Genoa, Italy; ' IRCCS Ospedale Policlinico \\ San Martino, Genoa, Italy; ${ }^{d}$ Neurochemistry Lab, Department of Clinical Chemistry, \\ Amsterdam University Medical Centers, Vrije Universiteit, Amsterdam, The Netherlands; \\ eKlinikum rechts der Isar, School of Medicine, Technical University on Munich, Munich,

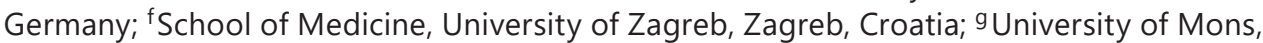 \\ Mons, Belgium; ' University Medical Centre Ljubljana, Ljubljana, Slovenia; 'University of

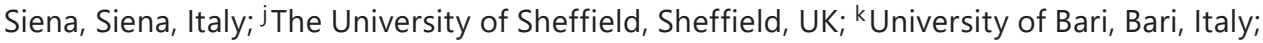 \\ 'National and Kapodistrian University of Athens Medical School, Athens, Greece
}

\section{What Is It about?}

Biomarker testing is improving the prospects for tackling Alzheimer's disease (AD) and other dementias, and holds the key to discovering new treatments. Early diagnosis and more personalised healthcare will have a central role to play in confronting this massive challenge to Europe's citizens and its healthcare systems. The EU is itself in constant evolution, both organically, as its competences are progressively refined, and in response to changes in the wider world. This paper discusses the challenges, lists the achievements to date, and highlights the actions needed to allow biomarker testing to deliver more fully on their potential in AD.

\section{Keywords}

Alzheimer's disease $\cdot$ Biomarkers · Diagnosis

\section{Abstract}

Alzheimer's disease (AD) and related dementia is one of the growing threats to the sustainability of health and care systems in developed countries, and efforts to find therapies have had scant success. The main reasons for this are lack of efficient therapy, which is linked to too late discovery of the disease itself. With this in mind, biomarkers are recognised as an element which can bring a major contribution to research, helping elucidate the disease and 
the search for treatments. They are also playing an increasing role in early detection and timely diagnosis, which are considered the principal hopes of effective management in the absence of an effective drug. The current arsenal of biomarkers could already, if more widely deployed, provide an effective minimum service to patients and health systems. A concerted action by policy makers and stakeholders could drive progress in access to AD biomarker testing to provide an optimum service in the medium term. This paper discusses how to improve the use of and access to biomarker testing in the detection and diagnosis of $A D$ and other diseases featuring dementia, and how EU healthcare systems could benefit. It outlines the challenges, lists the achievements to date, and highlights the actions needed to allow biomarker testing to deliver more fully on their potential in AD.

(C) 2020 The Author(s)

Published by S. Karger AG, Basel

\section{Introduction}

Among the many challenges to Europe's health systems, the most daunting include Alzheimer's disease (AD) and other diseases featuring dementia, because of their prevalence among an increasingly elderly population, and the continuing lack of effective therapies even to modify their progress. Numerous failed clinical trials have demonstrated the need to take into account early detection of disease, much before the onset of symptoms, and to explore personalised (precision) medicine treatment approaches. Dementia is a distressing and onerous burden on individuals - in Europe, an estimated 10.5 million people live with dementia, and this number is expected to increase to 18.7 million by 2050 . There are grave implications for society too, costing an estimated EUR 167.5 billion per year in the EU - or an estimated cost of EUR 22,000 per patient per year. AD is the most common type of dementia, affecting $50-70 \%$ of all patients, destroying irreversibly the cognitive abilities of affected people, and greatly diminishing their quality of life. Its impact in high-income countries is expected to double between 2015 and 2050, since more people living into older age means the number of individuals with dementia is increasing, and the economic, health, and social care costs of dementia are escalating [1]. Given the increase in healthcare spending required and loss of productivity - particularly among carers - due to neurocognitive disorders, use of and access to biomarker testing can have a substantial positive impact on future research and care costs. The urgency of finding treatments to modify the course of AD has driven additional public investment in Alzheimer's research in the USA and Europe, and the creation of innovative mechanisms such as the public-private Dementia Discovery Fund to invest in preclinical and early-stage clinical research [2].

However, despite the significance of $\mathrm{AD}$ and other dementias, there is still incomplete understanding of these conditions. Drug development for AD remains a high-risk endeavour. Significant medical and scientific advances in recent decades have not led to significant successes in research and development programmes on potential drugs for AD. There are currently no approved treatments to prevent, slow, or cure it, and only few candidates in latestage development. Some failures in clinical trials have indeed added to understanding about the disease itself and its pathology, but many challenges persist, including the diversification of potential targets for drug development, and the translation of research findings into clinical applications. Clinical development programmes in $\mathrm{AD}$ and other relevant neurodegenerative and central nervous systems diseases take longer, are riskier, and have lower regulatory success rates than in other indications - leading to very high development costs, attrition in subject compliance during lengthy studies, and limited therapeutic target diversity [3].

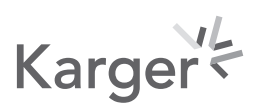


In these circumstances, biomarkers have a crucial role in helping pierce the fog that surrounds these diseases - and that blights the life of the patients who suffer from them. Biomarker testing offers hope of better understanding and diagnosing the disease, and of developing solutions and treatments that can slow the progression of $\mathrm{AD}$, easing suffering, and even, in time, restoring and protecting health [4].

\footnotetext{
What are biomarkers?

Biomarkers are measures of what is happening inside the living body, shown by the results of laboratory and imaging tests. Across a wide range of health conditions, from cancer to AD, they can help doctors and scientists diagnose diseases, find health risks in a person, monitor responses to treatment, and see how a person's disease or health condition changes over time. Some biomarkers may be used to identify or to rule out causes of symptoms for some people. They also play a central role in research, providing information on disease processes and on responses to therapeutic approaches, determining relevant and reproducible outcome measures [5].
}

Through the use of biomarkers, researchers can now see Alzheimer's-related changes in the brain while people are alive, diagnose, track the disease's onset and progression, and test the effectiveness of promising drugs and other potential treatments. In the clinic, biomarkers are central to a diagnostic assessment for people with symptoms of Alzheimer's - alongside a medical history, physical exam, laboratory tests, and a range of neurological and neuropsychological tests of mental functions. Different biomarkers provide different types of information about the brain and may be used in combination with each other and with other clinical tests to improve the accuracy of diagnosis - for example, in cases where the age of onset or progression of symptoms is not typical for neurocognitive disorders. Because patients progress at different rates, biomarkers may help predict and monitor their progression.

However, many hurdles exist (as outlined later). In summary, they range from the scientific to the contextual, from diagnosis to research, from concept to validation, and from the laboratory and the clinical trial to clinical practice. These obstacles need solutions, even for a minimum level of use and access across Europe. To fully realise the potential of biomarker testing in these diseases, a significantly improved context of use for the biomarkers in specific clinical setting and addressing different questions from physicians will need to be created (for details, see the section A Recipe for Optimum Testing, below). The limited availability and clinical use of diagnostic tools and services, including both cognitive and biomarker-based approaches, hampers the timely diagnosis of the disease, the development of effective therapies, and undermines long-term public health outcomes that can be realised [6] (Fig. 1).

\section{Minimum Testing}

The major hurdle is, of course, that we have very limited therapeutic options for AD. Most current research is focused on seeking treatments to delay, and perhaps prevent, the progress of the disease [7]. In this context, biomarkers are used to include patients with specific pathologies in the earliest possible stage, for example amyloid-positive preclinical individuals need to be selected in order to evaluate drugs targeting amyloid effectively. Likewise, amyloid- and tau-positive individuals should be selected for any drug addressing AD pathology specifically. In addition, biomarkers are needed to better understand the sequential and interacting pathological cascades, to identify individuals who are most likely to respond to a specific treatment in order to better "enrich" the targeted subject population of clinical trials, and to assess the safety and effectiveness (outcome) of potential therapies. Clinical practice increasingly

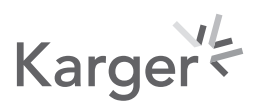




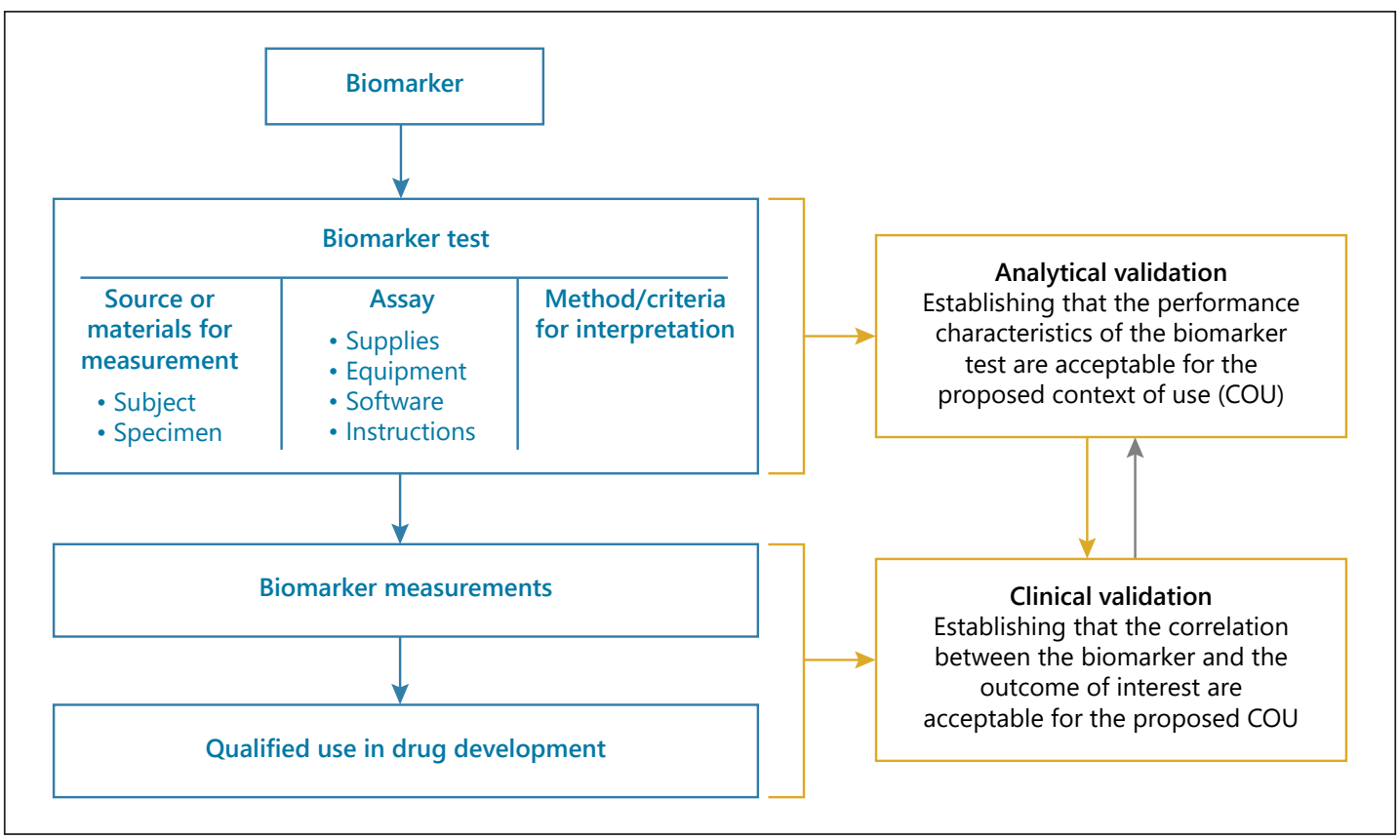

Fig. 1. Validation. The interrelated processes of analytical and clinical validation of the biomarker for its proposed context of use. Source: Alzforum [56].

focuses on early detection and diagnosis of AD in its early stages to improve the prospects for disease management. But it is also because potential treatments to delay AD are thought to be most effective when provided in the early stages of disease, before symptoms appear [8].

\section{Early Detection}

Changes in the brains of people with $\mathrm{AD}$ or dementia begin years before symptoms appear. Studies suggest that biomarker changes can be detected well before the onset of cognitive impairment symptoms. Greater understanding of the pathology and course of AD has led to it being re-conceptualised as a disease continuum, in which patients experience a long asymptomatic (preclinical) phase in which neuropathological changes occur but cognitive ability is normal, followed by a symptomatic (prodromal or predementia) phase of progressive cognitive decline before the onset of functional impairment and overt dementia [9].

Finding these changes early in the disease process helps identify people who are at the greatest risk of Alzheimer's or another dementia. This new concept of AD, with its associated diagnostic criteria, depends on the presence of biomarkers to identify preclinical states and prodromal stages of $\mathrm{AD}$. It requires evidence of both specific clinical features and in vivo biological evidence of an underlying abnormal pathology that is well defined and detected using biomarkers. AD is thus considered a clinico-biological entity that can be identified in vivo, and revised criteria for the clinical diagnosis of AD consider a symptomatic predementia phase of $\mathrm{AD}$, referred to as "mild cognitive impairment (MCI) due to $A D^{\text {" }}[10,11]$.

The 2018 research framework of the NIA-AA [12] clearly considered AD no longer a clinical diagnosis but a biological diagnosis, irrespective of the presence and severity of symptoms! Effective early intervention requires the identification of early-stage patients, by 
screening patients for signs of early-stage memory loss or MCI, then testing for the AD pathology or other dementia [13].

Even in the absence of any therapy able to reverse the disease, there are advantages to early detection and diagnosis at a time when people first seek help due to being worried about changes in cognition, behaviour, or functioning not necessarily resulting in dementia. This offers the opportunities of implementation of coordinated care plans, better management of symptoms, and patient safety [14]. Recognition of AD during the prodromal stage can allow physicians to offer therapies that address specific symptoms, such as anxiety or impaired sleep, and to manage medications prescribed for comorbidities that may be inadvertently exacerbating dementia or other emerging symptoms. Not only does biomarker use lead to the detection of $\mathrm{AD}$, but also to the exclusion of $\mathrm{AD}$ and detection of other pathologies causing cognitive impairment, which are not always immediately clinically evident. Timely diagnosis of $\mathrm{AD}$ has the potential to reduce the impact of no or a delayed diagnosis or misdiagnosis. It could also reduce costs later on in the disease process, as a well-informed patient is less likely to experience crisis situations or premature institutionalisation. Patients could put into place care planning in advance and make end-of-life decisions, consider changing unhealthy lifestyles, and seek better medical care. Furthermore, it could allow, in the future, access to interventions that would slow or lessen the disease process: as and when any disease-modifying therapy becomes available, early diagnosis will provide the opportunity to achieve added value from earlier treatment or intervention within a clinical trial before the onset of dementia [15]. The downsides of the early diagnosis is that: (1) we do not have a clear understanding of the time between identification of a preclinical or early stage and the onset of dementia, and (2) some of the subjects who test positive to biomarkers will not develop dementia during their lifetime.

Early detection and diagnosis and symptom management are central elements in a comprehensive response, along with access through clear regulatory pathways, manufacturing, and sustainable market schemes. With the impact of the increasing incidence of AD on individuals and society becoming clearer, the early diagnosis and appropriate treatment of affected individuals is a public health priority [8].

\section{The Current Arsenal of Biomarkers}

There is a growing arsenal of biomarkers - neuroimaging, cerebrospinal fluid (CSF), genomic, and peripheral systems, identifying relevant molecular/cellular abnormality - that can aid diagnosis and management, differentiate $\mathrm{AD}$ and other dementias, and play a role in research. The most widely used biomarkers measure changes in the size and function of the brain, and levels of specific proteins seen on brain scans and in CSF, and with a lesser accuracy, in blood.

Brain imaging, or brain scans, can measure changes in the size of the brain, identify and measure specific brain regions, and detect biochemical changes and vascular damage. In clinical settings, doctors can use brain scans to find evidence of brain disorders such as tumours or stroke that may aid in diagnosis. In research settings, brain imaging is used to study structural and biochemical changes in the brain in AD and related dementias. The principal imaging techniques - computerised tomography (CT), magnetic resonance imaging (MRI), positron emission tomography (PET) - are outlined below [16].

CT uses enhanced X-ray technology. A routine scan of only a few minutes can show the size of the brain and identify a tumour, stroke, head injury, or other potential cause of dementia symptoms. It can show shrinkage of brain regions that may occur in dementia, but cannot easily measure changes over time. This may be acceptable in the oldest patients with comor-

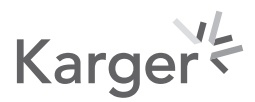


bidities in whom the likelihood of reaching a precise diagnosis is low, and in low-income countries where access to MRI is limited [17].

MRI uses magnetic fields and radio waves (for about $30 \mathrm{~min}$ for diagnostic purposes and up to $2 \mathrm{~h}$ for research purposes) to produce detailed images of the size and shape of the brain and brain regions. It can identify some causes of dementia symptoms, such as a tumour, stroke, vascular damage, or head injury, and may also show areas of brain atrophy, or whether abnormal changes are present. Repeat scans can show how a person's brain changes over time. Evidence of shrinkage may support a diagnosis of Alzheimer's or another neurodegenerative dementia, but cannot indicate a specific diagnosis. However, it can identify or rule out causes of memory loss, such as a stroke or other vascular brain injury, tumours, or hydrocephalus. Researchers use different types of MRI scans to study brain structure, chemistry, blood flow, and function, as well as the size of brain regions. In clinical trials, MRI can be used to monitor the safety of novel drugs and to examine how treatment may affect the brain over time [18].

PET, commonly used in dementia research, uses small amounts of injected radioactive tracers to measure specific activity in different brain regions over the space of an hour, revealing regions of normal and abnormal chemical activity. Fluorodeoxyglucose (FDG)-PET scans measure glucose use in the brain to support a diagnosis of a specific cause of dementia: sufferers often have abnormal patterns of decreased glucose use in specific areas of the brain. Amyloid PET scans (using florbetapir, flutemetamol, florbetaben, or Pittsburgh compound B as tracers) measure abnormal deposits of the protein $\beta$-amyloid: higher levels in the grey matter are consistent with the presence of amyloid plaques, a hallmark of Alzheimer's pathology. A negative amyloid PET scan rules out most forms of AD. Tau PET scans detect abnormal accumulation of the protein tau, which forms tangles in nerve cells in AD and many other dementias (with tracers including AV-1451, PI-2620, and MK-6240 currently under study in clinical trials and other research settings) [19].

In clinical care, FDG-PET scans may be used if a doctor strongly suspects frontotemporal dementia (FTD) as opposed to Alzheimer's dementia based on the person's symptoms, or when there is an unusual presentation of symptoms. FDG-PET is also useful as a supportive biomarker for DLB (whereas dopamine transporter [DAT]-SPECT is an indicative biomarker). These scans can also help diagnosis in the face of unusual or very mild symptoms, an early age of onset (under 65 years old), or any of several different conditions, such as severe depression, that may contribute to dementia symptoms.

In research, amyloid and tau PET scans are used to determine which individuals suffer from AD, to identify clinical trial participants, and to assess the impact of experimental drugs designed to affect amyloid pathways [5]. The same approach is under study for tau PET scans. Amyloid PET imaging is sometimes used by medical specialists to help with a diagnosis when $\mathrm{AD}$ is suspected but uncertain, even after a thorough evaluation. Other promising tracers are under development, mainly targeting brain inflammation and synaptic function.

CSF analysis can reveal changes in proteins and other substances produced by affected brain tissues whose levels change years before symptoms of Alzheimer's and other brain disorders appear. The most widely used CSF biomarkers for AD measure certain proteins: $\beta$-amyloid 42 (the major component of amyloid plaques in the brain), $\beta$-amyloid 40 , tau, and phospho-tau (major components of tau tangles in the brain). In $\mathrm{AD}, \beta$-amyloid 42 levels (alone or as a ratio to $\beta$-amyloid 40) in CSF are low, and tau and phosphorylated tau levels are high, compared with levels in people without AD or other causes of dementia [20].

In clinical practice, CSF biomarkers may be used to help diagnose Alzheimer's in cases involving an unusual presentation of symptoms or course of progression. $\beta$-Amyloid $(A \beta 42$, $\mathrm{A} \beta 42 / 40$ ), total tau ( $\mathrm{T}$-tau), and phosphorylated tau have a high diagnostic accuracy in early stages of AD. Many studies have shown that patients with AD have elevated levels of T-tau

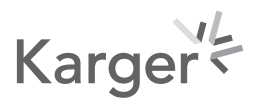


and P-tau protein and decreased levels of $\beta$-amyloid, and this is in fact the basis of the biological definition of AD. Other promising biomarkers in the CSF include TREM2, a transmembrane receptor that is expressed by microglia and visinin-like protein 1 , a neuronal calcium-sensing protein that participates in neuroprotection and neurotoxic processes in the brain. It is a marker of neural damage and it correlates well with T-tau and phosphorylated tau. Neurogranin (NGRN) is a postsynaptic protein that is expressed mainly in the cortical regions and is associated with cognition. Reduced NGRN levels are found in the cortex and hippocampal region of the brain, which are most affected by AD. NGRN can be used to predict the progression of cognitive deficit and can indicate the loss of synapses.

In research, CSF biomarkers are valuable tools for early detection of a neurodegenerative disease. They are also used in clinical trials to assess the impact of experimental medications. The $\beta$-site of APP cleaving enzyme 1 (BACE-1) is a $\beta$-secretase that is important for the cleavage of APP protein, while the resulting peptides aggregate and form extracellular plaques. The levels of this enzyme are elevated in people with AD compared with healthy controls and patients with other forms of dementia. Recently, BACE-2 has been reported as a protective enzyme against dementia, with huge potential to become a useful biomarker. Neurofilament light, a monitoring protein, and YKL-40, an inflammatory protein that is elevated in $\mathrm{AD}$, have evidence for use in clinical trials to monitor effects, with similar potency to NGRN [21].

Proteins that originate in the brain, such as tau and $\beta$-amyloid 42 , and whose levels can vary as a result of $\mathrm{AD}$, a stroke, or other brain disorders, may nowadays be measured with sensitive blood tests. Methods are being explored to improve their accuracy for identifying $\mathrm{AD}$ and related dementias, particularly for $\beta$-amyloid 42 , but these methods have not yet been employed in clinical practice.

Blood tests may be used in the future for screening and perhaps diagnosis, since many other proteins, lipids, and other substances can be measured in the blood. There is growing evidence of the value of phosphorylated tau in diagnosing AD [22-25].

Genetic testing is another consideration. Problems with genes can cause diseases like AD, and analysis of DNA from blood or saliva can determine a person's genetic makeup and reveal genetic combinations that may change the risk of developing a disease that causes dementia. Genetic mutations in presenilin or APP cause familial AD, which make up less than $1 \%$ of all cases. Variation in ApoE, the APoE4 genotype, is the major risk factor in sporadic AD. For instance, APOE $\varepsilon 4$ is the main genetic risk factor for late-onset AD. However, genetic testing does not identify other genetic and environmental factors, lifestyle choices, and family medical history that affect a person's risk of developing AD. A genetic test typically provides accompanying genetic counselling before the test and when the results are received to discuss risks, benefits, and limitations of the test results with the patient.

Genetic tests are not routinely used in clinical settings to diagnose or predict the risk of developing Alzheimer's or a related dementia. However, a neurologist or other medical specialist may order a genetic test in rare situations, such as when a person has an early age of onset or a strong family history of Alzheimer's or a related brain disease. In research studies, genetic tests may be used, in addition to other assessments, to predict disease risk, help study early detection, explain disease progression, and study whether a person's genetic makeup influences the effects of a treatment [5].

Stem cells offer new possibilities in early diagnostics. Thus, it is possible to use stem cell technology to obtain neurons from almost any cell in the human body. One approach is the transformation of hair follicles into neurons, which can then be used either for early diagnostics or for discovering new pathophysiological elements of AD. Similar application of stem cells for early diagnostics is present in the "Check My Brain" diagnostic and therapeutic platform [26]. It offers a simple procedure which provides three sets of information:

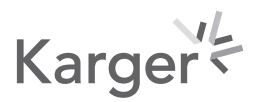


(1) early diagnostics of brain diseases, by detecting biomarkers using cell cultures, (2) the prognosis of disease progression by assessing the changes in biomarker levels, and (3) personalised instructions on dietary, supplementary, and other approaches that could bring benefit for the individual brain cells. By combining disease detection much before the onset of visible symptoms and the individual-tailored approach, "Check My Brain" points the direction that is required in addressing the threat of neurodegenerative diseases.

Exosomes can also be used in the diagnostics of brain diseases. The detection of exosomes in body fluids is becoming a novel tool for improving diagnosis and monitoring the biological activity of pathological processes before the manifestation of apparent clinical symptoms. Indeed, toxic proteins present in the exosomes can be detected in the early stage of a variety of neurodegenerative diseases $[27,28]$.

\section{Hurdles}

To exploit the current range of biomarkers in the clinic or in research, changes are needed to increase their use and access: there are challenges to be met in areas as distinct as validation of their value, standards, regulation, payment, take-up, trial organisation, ethics, social policy, and communication. Standardisation is needed, both in terms of validation and usage. First steps have been made to produce new scientific standards to calibrate diagnostic tools for more accurate diagnosis and detection of $\mathrm{AD}$, such as reference material of $\beta$-amyloid proteins by the European Commission's research testing centre.

There is not enough cross-sectoral collaboration and interdisciplinary research to advance understanding of how $\mathrm{AD}$ develops and to translate emerging knowledge more efficiently into preventive and therapeutic approaches. Governments do not invest enough in basic and applied science in order to understand the complex pathologies of dementia. In addition, in the healthcare systems that should be taking advantage of these innovations, there is a lack of coordination across specialisms and institutions, and often insufficient dialogue between specialists and primary care physicians to ensure wide awareness of the value of biomarker testing. What is urgently needed for more efficient introduction of biomarkers for brain diseases in everyday practice is more substantial funding of crossborder and multidisciplinary projects which will not only support basic testing, but also advance stage projects aiming to validate biomarkers.

Biomarker research is not integrated enough into clinical medicine, so its use often does not extend beyond clinical trials. There are wide gaps between the worlds of research and the clinic, where knowledge about the potential of biomarker testing - and experience and accumulated expertise - are lacking outside specialist centres. The relative merits of multiple pathway testing against single pathways and the value of precise diagnostic knowledge are not widely understood. In addition, research has tended to overlook the value of different biomarkers across the age spectrum, with most studies conducted in populations aged in their 50s, while most clinical cases are at 75 years or above.

In general, there is insufficient awareness of the concept that $\mathrm{AD}$ can be diagnosed by biomarkers - even in the most prosperous countries and most sophisticated health systems in Europe. Equally, there is little familiarity with the capacity of biomarkers to distinguish AD from other dementias.

For the same reason, biomarkers do not become important tools for regulatory decisionmaking. The stakeholder community fails to come together to enhance understanding of AD generally, as well as current and possible future treatment options, or to develop plans that will secure continuous progress in basic science and bring innovations to markets. There is

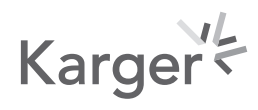


growing interest in creating academic-industry partnerships in areas that could lead to the co-development of drugs and biomarkers [26].

The use of biomarkers in clinical settings is limited at present, particularly in primary care, where there is limited experience. While neurologists, geriatric psychiatrists, and geriatricians more frequently order biomarker tests, there is a lack of familiarity among general practitioners, and a lack of expertise in interpreting the results. Although MRI and CSF biomarkers are already widely used in clinical practice, even for these tests, a lot of work still needs to be done to translate research findings to daily practice as clinicians vary greatly in their knowledge of these markers. Questions still hover over the consistency of interpretation of evidence before recommendations for adopting new technologies are made in clinical practice. This often translates into a vicious circle of underuse, so that there are wide variations in the availability of services for testing across Europe and across its regions, skewed in terms of cost and accessibility - notably in low- and medium-income countries.

Translating emerging tools for early diagnosis into clinical practice requires close collaboration between innovators, policymakers, and the payer community. For amyloid PET, whose use is self-evident with respect to clinical trials in $\mathrm{AD}$, clinical utility still has to be clearly established [29], although some studies now document the change in diagnosis and management of patients after performing amyloid PET. The decision for clinicians of whether to initiate diagnostic testing and choosing a test is still quite novel, so it is not yet common practice to involve patients and their caregivers in their diagnostic dilemmas. Despite a wealth of literature on AD biomarkers, there is a gap between the published value and the actual utilisation of biomarkers in daily clinical practice. The availability of biomarker tests poses the challenge for the clinician to select the right tests for each patient. In addition, effectively communicating with patients and deciding mutually whether to use certain tests is difficult, especially in view of the cognitive deficits that come with (prodromal) AD. The need for early detection and treatment, while a hot topic in clinical research, has not yet translated into a change in clinical practice, or into payer and policy maker actions. As a result, the patient journey remains unnecessarily long and arduous. In clinical practice, diagnosis often occurs late if it happens at all [30].

The quantification of validity in different settings would be useful (tertiary centres, population-based general practice) given that biomarker use is largely limited to tertiary research centres. The predictive value of different settings may be very different.

The problem is exacerbated by regulatory and payment difficulties. Regulators base decisions primarily on risk/benefit analysis, and in AD the measurement of clinically meaningful benefit has proved challenging. Some diagnostic biomarker tools that detect $\beta$-amyloid deposition through PET imaging or CSF analysis have received regulatory approval but not widespread payer support. Many health insurance plans cover only certain limited types of biomarker tests for dementia symptoms, and their use must be justified. Payers often do not understand the value of potential treatments, and remain on the sidelines as spectators rather than giving active support for innovation. Current reimbursement systems are frequently opaque over when and on what conditions - and at what cost - biomarker tests are reimbursed. Even hospital specialists comment on the tendency for hospitals to seek trade-offs in agreeing reimbursement, requiring a corresponding reduction in other costs. This problem is compounded when businesses are insufficiently transparent about the cost of the therapies they are offering or unrealistic regarding the price demanded [31].

There are also unresolved questions over the accuracy or aptness of some techniques in clinical practice. Back in 2015, Cochrane reviews of CSF biomarkers suggested that there was not sufficient evidence to support the routine use of CSF $\beta$-amyloid or PET in the diagnosis of $\mathrm{MCI}$ due to $\mathrm{AD}$ and other dementias [32]. The use of biomarkers as diagnostic criteria for $\mathrm{AD}$ might be valuable in research, they said, but they noted concerns about the premature use of such criteria beyond the research setting [33].

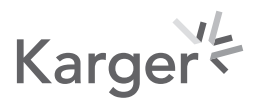


Since then, however, several guidelines/recommendation/criteria have been published, including on appropriate use criteria for lumbar puncture and CSF testing in the diagnosis of $\mathrm{AD}$, and disease biomarkers in the diagnostic evaluation of $\mathrm{MCI}$ [34-36]. Again, the setting is very important because there is a variation of the probability of diagnosis based on a priori probability, given by the prevalence of dementia in different settings.

Even where there are alternative measures rapidly upcoming, such as correlations of biomarker concentrations in patients and controls, these may indicate the potential value of a marker in the research phase, but there is not yet information about how the test will perform in a typical clinical setting. More definitive evidence from studies done in consecutive series of presenting patients is needed before these biomarkers can be recommended for routine clinical use, they say.

Developing blood biomarkers for AD has proven difficult; while the CSF is continuous with the brain extracellular fluid, with a free exchange of molecules from the brain to the CSF, only a fraction of brain proteins enter the bloodstream. Blood is also a more challenging matrix than CSF for brain biomarkers. The minute amounts of brain proteins entering the blood have to be measured in a matrix containing very high levels of plasma proteins, such as albumin and IgG, introducing a high risk of interference in analytical methods. In addition to dilution, brain proteins released into blood may be rapidly degraded by proteases, metabolised in the liver, or cleared by the kidneys, which will introduce a variance that is unrelated to brain changes and difficult to control for. Nevertheless, technical developments in the field of ultrasensitive immunoassays and mass spectrometry have given new hope [37]. The position of a blood-based biomarker is a prescreening tool, to reduce the number of lumbar punctures to collect CSF and to reduce the cost of PET scans [25].

Prevention studies using biomarkers as entry criteria and/or as efficacy variables do have limitations, such as the costs of screening for suitable candidates, relative invasiveness of the procedures (PET and lumbar punctures), access to the technology (PET, 3-T MRI), and the potential lack of utility in the most common late-onset dementias with multiple co-morbidities. These issues all need resolving in order to provide minimum testing.

Meanwhile, the arsenal is being developed, and the prospects are opening up for an even greater contribution of biomarkers to tackling AD. For dementia with Lewy bodies (DLB) and FTD, which cover at least the $30 \%$ of all dementias, biomarkers are also emerging. DATSPECT, MIBG cardiac scintigraphy, and polysomnography are indicative biomarkers for DLB, while the research to show $\alpha$-synuclein in CSF, in skin or salivary gland biopsy, or in nasal mucosal brushing with rtQuic technology is ongoing. Research is also underway to find an $\alpha$-synuclein PET tracer. For FTD and TDP-43, progress is less advanced, but serum progranulin dosage in progranulin mutants has already been realised, and genetic for TARDP, c9orf72, progranulin, and FUS mutation also merits attention [38].

\section{Future Arsenal}

Improved diagnosis and more productive research are in reach, through better understanding of the risk factors and genetic variants involved in $\mathrm{AD}$, and through sharper identification of patients or of trial participants with certain genes or amyloid levels. A full arsenal of validated and qualified biomarkers will help the refinement and harmonisation of criteria of $\mathrm{AD}$ across all stages in order to ensure a better definition of patient populations in clinical trials. Researchers are studying other biomarker tests for possible use in diagnosing and tracking $\mathrm{AD}$ and other types of dementia, including reduced ability to smell, the presence of certain proteins in the retina of the eye, and other proteins that indicate the health of neurons.

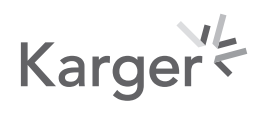


Researchers are also working on developing and validating a full range of biomarkers, particularly those that are less expensive and/or less invasive, to help test drugs that may prevent, treat, and improve the diagnosis of AD and related dementias. Here, one of the aims is to use biomarkers in combination to build a model of AD progression over decades, from its earliest, presymptomatic stage, through to dementia [39].

There are some promising fields opening up. Amyloid proteins and pTau181 can now be measured in blood, showing clear differences in AD patients from controls. Tau protein in CSF has been found to be present as truncated fragments, and it is possible that development of assays based on antibodies for specific tau fragments will improve performance. Measurement of T-tau or P-tau in neuron-enriched exosome preparations may improve performance for tau as a blood biomarker, although further studies are needed to validate this finding. Neurofilament light in plasma could become a screening test at the first clinical evaluation - even in primary care - of patients with cognitive disturbances, since it has now been established that high plasma (or CSF) neurofilament light chain (NFL) is not specific for AD, but instead is found in many neurodegenerative disorders, such as FTD, progressive supranuclear palsy, and corticobasal syndrome. Plasma NFL has the potential to be a simple, non-invasive, and cheap screening tool, primarily to rule out neurodegeneration [5].

FDG-PET may be used in MCI for obtaining an onset-related estimate of the time free from disease. It also appears to be the only significant predictor of cognitive decline in early-onset patients, as well as to rule out a neurodegenerative disease as a cause of those cognitive symptoms.

Since amyloid build-up appears to precede cognitive decline by many decades, it allows for primary prevention studies in higher-risk populations defined by PET scans or CSF amyloid protein analysis. This build-up correlates to some degree with the apoE4 genotype, another biological marker for risk of $\mathrm{AD}$ that is being used as inclusion criteria in prevention studies. Biomarkers of disease progression, such as brain atrophy using MRI, regional hypometabolism using PET with ${ }^{18} \mathrm{~F}$-glucose, or elevation of levels of tau and NFL in CSF, can be used as efficacy variables in prevention studies, particularly in proof-of-concept or as evidence for target engagement in phase II studies [37] (Fig. 2).

The last 20 years have seen an enormous expansion in research on fluid biomarkers for AD. The core CSF biomarkers have been evaluated in hundreds of clinical neurochemical studies with extraordinary consistent results, showing high diagnostic accuracy both for AD dementia, but also for prodromal AD. These biomarkers have undergone a phase of standardisation and new assay versions on fully automated instruments show excellent analytical performance. The core AD biomarkers are today part of research diagnostic criteria, it is possible to foresee an increased use of these diagnostic tests in clinical routine practice. The AD CSF biomarker toolbox has been expanded with novel biomarkers reflecting additional aspects of $\mathrm{AD}$ pathology, such as synaptic dysfunction. Validated biomarkers here include DAT-SPECT and MIBG cardiac scintigraphy, and further validated assays reflecting other pathologies common in age-related neurodegenerative disorders, for example Lewy body and TDP-43 pathology, could reach the stage of clinical applications in the coming years, so that CSF biomarkers can be part of a personalised medicine approach to the clinical evaluation of patients with cognitive disturbances. Blood biomarkers may eventually be implemented as screening tools in the first-in-line clinical evaluation of this group of patients. Since blood is more accessible than CSF, there is little doubt that blood sampling would be preferable to CSF when it comes to taking fluid samples to measure AD biomarkers, both for clinical diagnosis or screening and for repeated sampling in clinical trials [40]. The developments in imaging biomarkers (amyPET, Tau PET, DAT-SPECT, etc.) have been commented on elsewhere in this paper. In addition, an EEG (electroencephalogram) biomarker is a non-invasive and low-cost tech-

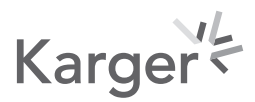




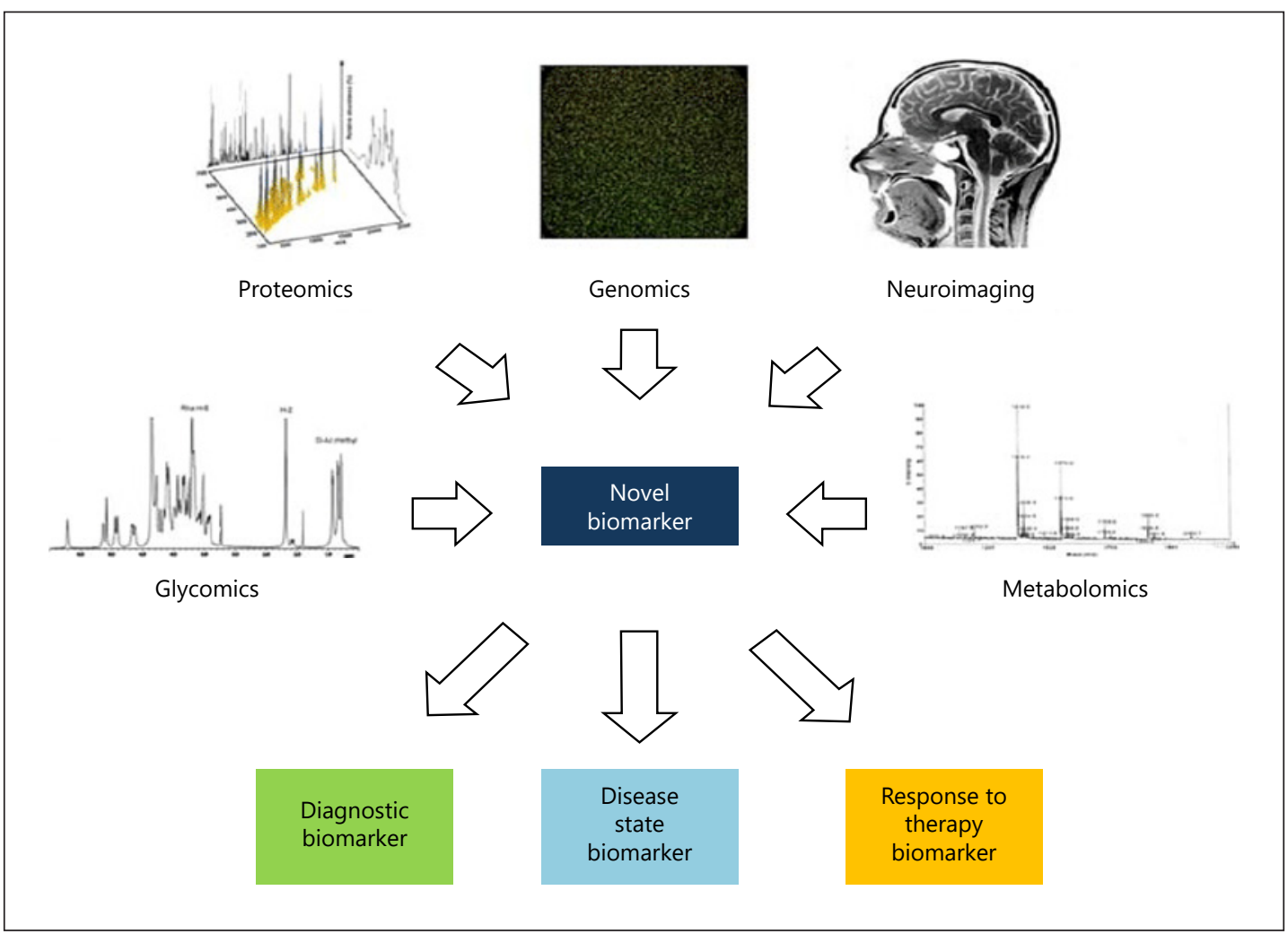

Fig. 2. Flow chart showing multifaceted approach towards biomarker development. Source: Gotovac et al. [57].

nique which could be used in combination with other techniques to highlight brain dysfunction typical of AD. Powerful AI tools have been developed to analyse functional connectivity and allow big data-based classification.

\section{Conditions for Further Progress}

Progress depends not just on science but on many contextual factors. There is a need for agreed biomarkers, prioritisation, guidelines, adequate funding, innovative and collaborative management, collaboration and planning for access, major improvements in trial organisation, understanding among regulators and payers, wider take-up, and addressing ethics/ social/communication issues.

Despite major advances in biomarker validation and partial success in qualification, science has still to develop sufficient means to identify all relevant molecular mechanisms or signalling pathways that could serve as biomarkers for the development and progression of neurological disorders. Significant progress in biomarker validation and qualification will only be possible with a more in-depth understanding of the relevant molecular and biochemical underpinnings of disease. The development of companion diagnostics would be a significant, but not complete, step towards understanding, diagnosing, and treating these diseases over the medium term. A major goal in biomarker research is to identify class-specific markers that could increase the efficiency of diagnosis and drug testing. However, to take advantage of biomarkers at the clinical level, strengthened academic-industry partnerships are needed for 
the co-development of drugs and biomarkers. The positive impact of validating multimodal biomarkers and imaging technologies on the acceleration of drug development processes cannot be overestimated [29].

Detecting amyloid deposition through ocular scans and blood markers may be useful as a screening tool - but development of tools to monitor disease progression (e.g., biomarkers to measure Tau or oxidative damage and inflammation) is strongly needed [41]. Researchers are studying many types of biomarkers that may one day be used more widely in doctors' offices and other clinical settings, but a key question is how can the potential of biomarkers move from patient group differences in clinical development to individual differences in precision medicine.

The advantage of predictive models is that they make it possible to estimate, given socio-demographic and clinical features, the patient-specific time-to-progression.

This in turn highlights a potential problem at the level of infrastructure in the event that a therapy becomes available to delay disease progression. Provision at scale of such a treatment in a preventive treatment paradigm implies the need to screen, diagnose, and treat a large population of patients with $\mathrm{MCI}$ as and when a therapy first becomes available. Treatment for these patients should be provided as quickly as possible to prevent progression to full-blown Alzheimer's dementia. However, a study of the healthcare system infrastructure in the six most prosperous European countries revealed gaps in preparedness to evaluate, diagnose, and treat the expected number of patients. The capacity for biomarker testing itself would not be the principal constraint, but rather the delays in the necessary visits to dementia specialists and the treatment of what is likely to be an infusion. Projected peak wait times range from 5 months for treatment in Germany to 19 months for evaluation in France. It could take until the middle of the century for wait times to disappear in some countries notably the UK and Spain - with more than 1 million patients with MCI progressing to Alzheimer's dementia while on waiting-lists in these six countries. The consequence for patients awaiting diagnosis and treatment would be continued progression of their disease to a later stage, at which point the treatment would no longer be effective. There are hopes that swifter diagnostic testing, through low-cost prescreening via blood analysis and upcoming uses of CSF biomarkers, could contribute to a solution. With that, 7 million people with $\mathrm{MCI}$ in the six European countries could seek timely diagnosis by a specialist and, when indicated, testing for Alzheimer's pathology to determine their eligibility for treatment. The analysis suggested that the healthcare systems in some of the European countries have insufficient capacity to diagnose and treat the large number of patients with early-stage AD. Complications of reimbursement, regulatory, and workforce planning would compound the difficulties [42].

This study also revealed a shortage of specialists, which is expected to worsen with the aging populations, and wide disparities in national processes for diagnosing cognitive impairment and dementia. Diagnoses may be conducted by neurologists or geriatricians in memory clinics or private neurology practices in France and the Netherlands, while in Sweden neurologists are typically not involved in diagnosis, which is typically conducted by geriatricians and psychiatrists.

Psychiatrists have distinct roles too. In Germany and Sweden $60 \%$ of them would be involved in formal diagnosis of $\mathrm{MCI}$ due to $\mathrm{AD}$, whereas the remaining $40 \%$ primarily practice psychotherapy. In contrast, in the UK, old-age psychiatry is a relatively small subspecialty, representing about $15 \%$ of total psychiatrists [43, 44].

Other initiatives for smaller member states are forming national referral centres, which could be coordinated between themselves and will have very clear standards in setting diag-

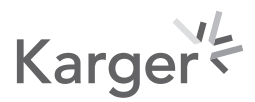


nostic rules. This could be supported through coordination funding at the EU level. This would support neurologists or psychiatrists who need to be updated on all the latest publications as well as setting guidelines.

\section{There Are Some Solutions}

The "Alzheimer's biomarkers in daily practice" (ABIDE) project is designed to translate knowledge on MRI, CSF, and PET diagnostic tests to daily clinical practice with a focus on MCI. The example of HIV/AIDS has shown that parallel pursuit of agreed biomarkers, prioritisation, adequate funding, innovative and collaborative management, collaboration, and planning for access can maximise speed and impact [30]. The collaborative experiences in seeking responses to the COVID-19 pandemic offer lessons in the merits of breaking out of traditional silos in the search for more effective healthcare.

Greater knowledge about biomarker testing in general, and the potential in AD, could promote acceptance and transform the approach among the general public, patients and carers, clinicians, regulators, and policymakers. A large European longitudinal study promoted by the EU or by a consortium of scientific societies (such as EAN or EANM) and co-funded by industry with an arm diagnosed with biomarkers and a control arm diagnosed with traditional means (i.e., neuropsychology + MRI) could provide valuable information on the use of biomarker-based diagnosis.

\section{There Are Some Conditions}

Opening and improving these diagnostic and treatment pathways will become increasingly important as new and earlier disease-modifying therapies are developed. Stakeholders from across the clinical community, healthcare providers, patients/patient organisations, governments, policymakers, and industry must work to enhance understanding of AD generally, as well as current and possible future treatment options. Such measures will be critical to ensuring patients can access biomarker testing, allowing future therapies to reach patients at the stage of disease progression when treatment can be most impactful [45]. Health systems should recognise their responsibility for improving as well as providing healthcare services.

Strenuous action must be taken to bridge the gaps between the research and clinical worlds, and to improve awareness of the merits of biomarker testing. This will depend on bringing greater focus to the challenging issues. The crucial task of promoting greater use of biomarker testing could be more effective if it concentrates initially on a handful of the most understood tests, to gain wider currency for the concept right across Europe. Education of and communication with primary physicians could be led by a group of early adopters, and a regulatory framework should be put in place to include biomarker testing in guidelines on clinical pathways. The framework should also provide endpoints agreed with all stakeholders, to provide some clarity of what is reimbursed and when and under what conditions, to influence payers and health technology assessment bodies to greater readiness over reimbursement.

Cross-sectoral collaboration and interdisciplinary research will be necessary to advance understanding of how AD develops and to translate emerging knowledge more efficiently into preventive and therapeutic approaches. Governments will need to invest to drive comprehensive efforts in basic and applied science in order to better understand the complex pathologies of dementia. Clinicians will need to engage with, support, and understand the next

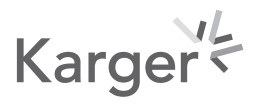




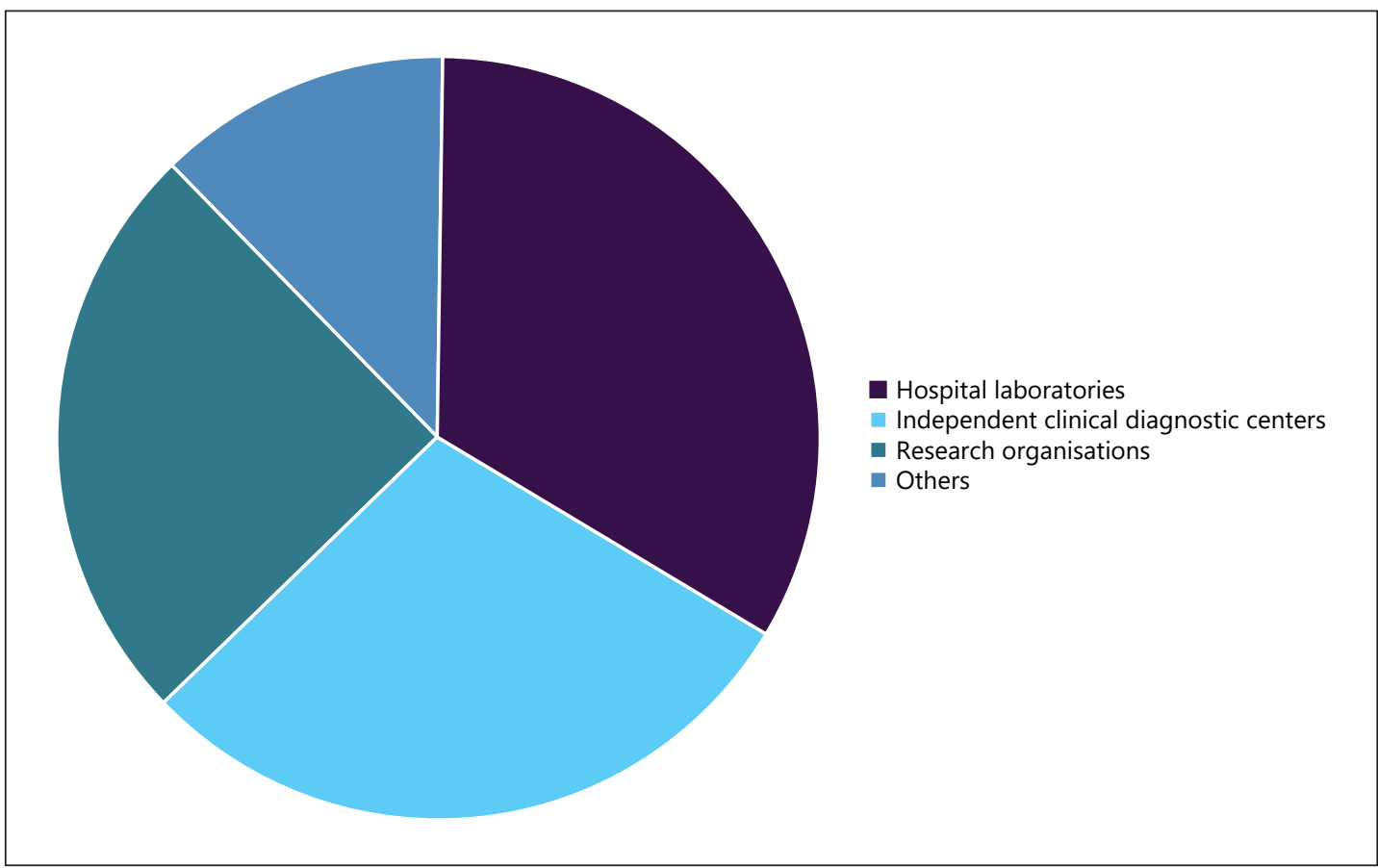

Fig. 3. Share of global neurological biomarkers market, by end-use, 2018. Source: Grand View Research [58].

generation of Alzheimer's medications. Payers will need to understand the value of potential treatments, moving from an observational role to active support for innovation. Businesses will need to be transparent about the cost of the therapies they are offering and to be realistic as regards the price. The whole community needs to come together to develop short- and long-term plans that will enable: continuous progress in basic science, translation of patient preferences into mutually accepted outcome measures, and the bringing to market of innovations (Fig. 3).

There is a need to integrate biomarker research into medicine - expanding the use of biomarkers beyond clinical trials, to become important tools for regulatory decision making and commercial diagnostics. At present, there is a lack of globally accepted and operationalised definitions of preclinical and early $\mathrm{AD}$, and this has a negative impact on regulatory policy development. Disparate views between innovators, payers, governments, businesses, and societies on what constitutes therapeutic and diagnostic value creates regulatory and access uncertainty [46].

The attention to securing a timely diagnosis also demands attention to some of the potential downsides of early information. They include stigma - public stigma, self-stigma, which may deter individuals from seeking professional help, or family/caregiver stigma - all of which can negatively affect the quality of life of people with dementia and their caregivers. There is also a suicide risk [29]. There is a lack of training for healthcare professionals, accompanied by diagnostic uncertainty, shortage of specialised diagnostic services, and the reluctance of healthcare providers to make a diagnosis when no effective disease-modifying options are available. Another concern is misdiagnosis, which can lead to inappropriate treatment of patients who could take unnecessary medications for AD or not receive correct therapy for potentially treatable disorders, similar to other diseases. The monetary costs to society of establishing systems for timely diagnosis and intervention may also be burdensome. 
Consequently, early diagnosis continues to be the exception rather than the norm, with less than half of people with AD actually diagnosed [8].

Few published studies have explored the advantages or risks of a timely diagnosis of AD, and more investigations are needed to confirm its feasibility and to evaluate the benefits and address the barriers that may impede it. Achieving a better performance would also require primary care physicians to be attuned to the early symptoms of AD. Clinical practice guidelines would need to be modified, and healthcare professionals, stakeholders, and policy makers would need to take action to fill gaps in knowledge, skills, attitudes, and resources [47]. Change in practice and attitude towards early diagnosis plays a key role here to ensure that we tackle this present and future healthcare burden [48].

Many infrastructure gaps will need to be filled to take advantage of innovation. Health system capacity would need to be expanded to cope with a widened range of testing and the implications for care and treatment that would result. Healthcare professionals would need education and training on the use of biomarker testing in neurological conditions. Payment arrangements for the conduct of testing and analysis and for reimbursement of the biomarker tests themselves would be needed. At present there is a disconnect between biomarker and therapy approval processes [42].

Other barriers to advancing innovative therapies for AD include lack of investment. Funding gaps arise from insufficient partnerships between governments, private, and philanthropic investors and inadequate investment in preclinical and translational research. There is often an accompanying reluctance to take a concerted approach to strengthening basic research and to sharing financial risks during early clinical development. However, without payers recognising the crucial value of fluid biomarker analysis and $\beta$-amyloid imaging in this devastating disease the incentives for investment and spending resources will vanish [29].

Clinical trials suffer from a lack of coordination. Recruitment remains a major bottleneck due to difficulties in identifying and characterising the right volunteers at an economically sustainable cost. Delays in trial recruitment hamper research progress, threaten internal uniformity and consistency, raise concerns about the reliability and generalisability of results, and increase costs. Inadequate links between the research community and people living with dementia lead to incomplete information about the needs and expectations of patients in clinical research, diagnosis, and symptomatic treatment.

There are insufficient - and insufficiently aligned - guidelines on which AD test to use, in which setting, for which patient, and how to weigh and communicate biomarker results to patients. Currently, healthcare providers' limited understanding of AD and treatment options fosters a passive approach to the disease in the medical community. Combined with social stigmas, this approach often leads to extensive delays in the diagnosis of $\mathrm{AD}$, and can preclude effective subsequent discussion of options with patients [49].

Much of the literature outlining the benefits of timely diagnosis of $\mathrm{AD}$ is based on expert opinion rather than research evidence. The scarcity of published studies assessing the benefits and challenges of timely diagnosis could be due to the fact that the definition of $\mathrm{AD}$ as an entity that encompasses both predementia and dementia phases is relatively recent and still not widely accepted [8]. Similarly, many studies of the possible economic benefits of early diagnosis and treatment of AD are not evidence-based but based on models that estimate the costeffectiveness of different theoretical interventions and/or outcomes. New research criteria still require further validation, and greater efforts are needed to harmonise the different clinical diagnostic criteria for AD. To translate emerging tools for early diagnosis into clinical practice requires close collaboration between innovators, policymakers, and the payer community [50].

\section{Karger'₹}


Horgan et al.: Biomarker Testing

\section{A Recipe for Optimum Testing}

European citizens and European society deserve a better-performing approach to tackling this devastating disease. It is not enough merely to cobble together a few short-term responses to the most immediate problems in providing an adequate testing service and in driving forward the research necessary to deliver solutions to AD and dementias. Europe deserves an optimum testing regime for the benefit of patients and their carers, but also to maximise the chances of addressing $\mathrm{AD}$, and of thereby easing the growing strains that dementia otherwise increasingly places on health and care systems.

In an optimum scenario, funding gaps would be addressed through partnerships between governments, industry, and private and philanthropic investors. Investment gaps in preclinical and translational research would be closed through a concerted approach to strengthening basic research and sharing financial risks during early clinical development. Significant progress in biomarker validation and qualification will be possible with a more in-depth understanding of the relevant molecular and biochemical underpinnings of AD.

Scientific progress would be aided by improvements to the evidence base for biomarker testing, and by initiatives such as the Integrated Development Initiative to increase the efficiency of clinical trials in AD through an international coalition of regulatory agencies, or through initiation of FDA-EMA interagency dialogue and qualification advice with sponsors to enable efficient parallel submissions for drug biomarker qualification or clinical outcome assessment qualification [50]. Flexible and more efficient trial designs would be developed, such as Adaptive POC Platform Trials or the IMI-EPAD project, for parallel evaluation of multiple treatments and combinations of treatments. Deep and frequent phenotyping cohort studies would be conducted to fill knowledge gaps and to complement volunteer registries for large proof-of-concept trials. Projects would be undertaken to harness the power of big data, led in the EU by the Innovative Medicines Initiative [51], and in the USA by the Global CEO Initiative on Alzheimer's Disease [52].

Advances in the understanding of the progression of dementia at the cellular and molecular levels would spur new research approaches. New technologies would facilitate diagnosis of the disease and development of drugs for dementia. Better understanding of the relationship between a class of drugs and a biomarker will make it possible to identify the patients most likely to benefit from treatment at increasingly earlier stages of the disease. Early and frequent interaction between industry and regulatory bodies will ensure studies are appropriately designed and biomarker test performance is well characterised [53]. This is coupled with placing an emphasis on undertaking a pilot of population-based studies so as to improve the testing validation process.

Information and education would be widely available for the public and professionals, to avoid misperceptions about the disease, treatment, and diagnosis. Better links would be established between the research community and people living with dementia to ensure complete information about the needs and expectations of patients in clinical research, diagnosis, and symptomatic treatment [3]. Physicians with expertise in AD and related dementias, neurologists, geriatric psychiatrists, neuropsychologists, and geriatricians would cooperate with regulators and health authorities in the development of standardised guidelines for testing and for interpretation of results, and laboratories would operate on standard protocols to ensure uniformity, permitting the establishment of a wide range of validated biomarkers. The qualification and validation of comprehensive exploratory and candidate biomarkers in AD is a key driver of current research programmes in academia and the pharmaceutical industry, essential to accelerate the development of innovative diagnostics and therapies [29].

There would be globally accepted and operationalised definitions of preclinical and early Alzheimer's to inform regulatory policy development. Views would be aligned between inno-

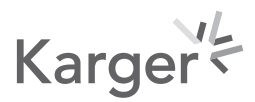




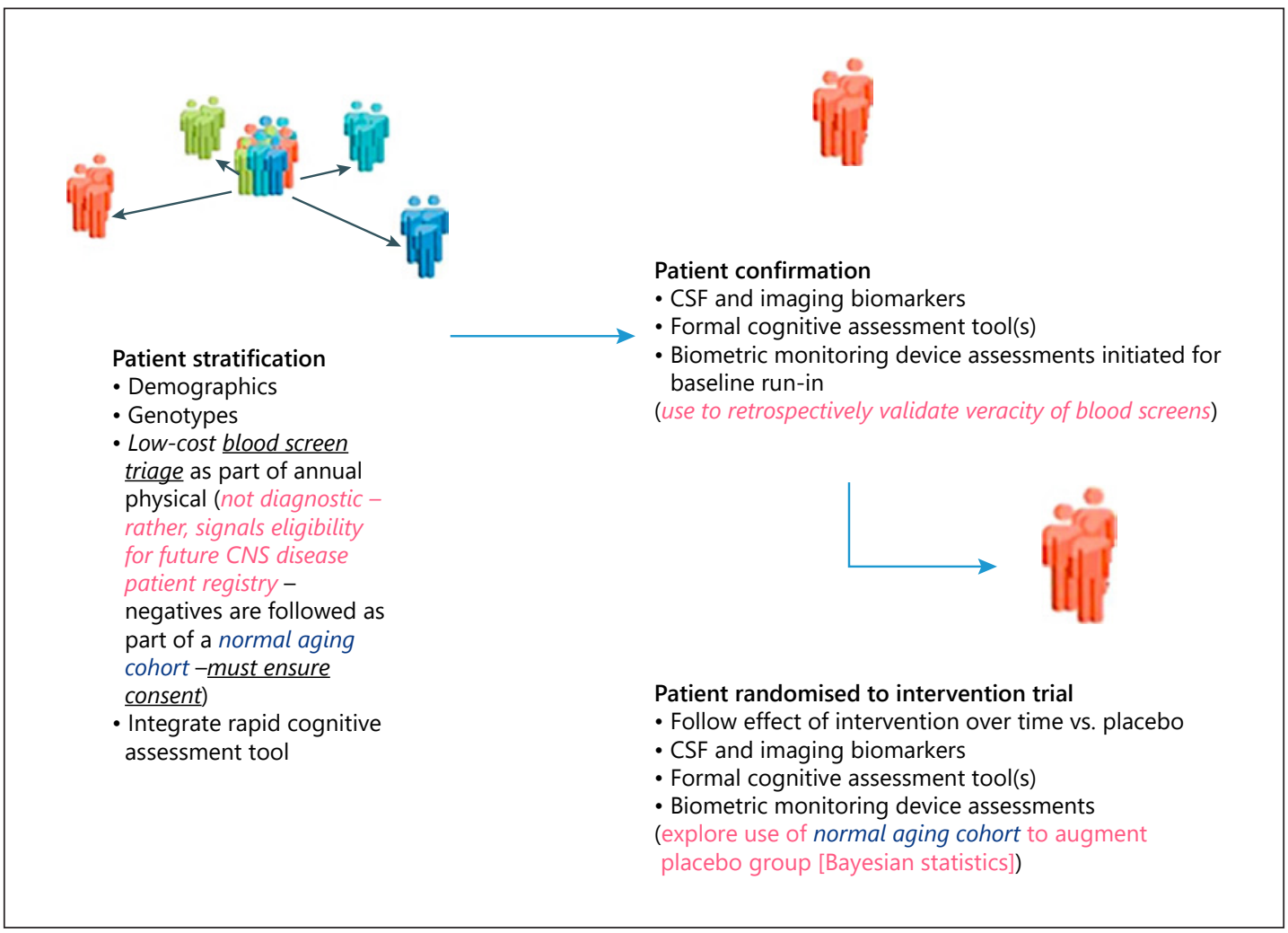

Fig. 4. Overarching biomarker strategy for CNS diseases. Source: Arnerić et al. [59].

vators, payers, governments, businesses, and societies on what constitutes therapeutic and diagnostic value, to alleviate regulatory and access uncertainty. Health insurance plans would take a more supportive role in the use of biomarker tests for dementia symptoms, on the basis of clear and transparent criteria [8]. Coverage and payment decisions for any future diagnostic and therapy for AD and other dementias would be based on medical evidence of positive health outcomes and relative costs of existing responses. CSF biomarkers for AD have been used in clinical practice and reimbursed by the health authorities in several countries, such as Sweden, the Netherlands, and Germany, for more than 10 years, based on rising demand from clinicians for these tests, helping to determine the true value of diagnostic testing.

Technological innovation in identifying low-cost, non-invasive markers will also help to further reduce the current costs for a baseline set of biomarkers for AD, including the partitioning of the cost of testing at baseline [33]. This would provide optimum testing (Fig. 4).

\section{Conclusion: A Fundamental Shift in AD Healthcare Strategy}

Biomarker testing is improving the prospects for tackling AD and other dementias, and holds the key to discovering new treatments. Early diagnosis and more personalised healthcare will have a central part to play in confronting this massive challenge to Europe's citizens and its healthcare systems. 
The EU is itself in constant evolution, both organically, as its competences are progressively refined, and in response to changes in the wider world. In healthcare, its evolution is marked not only by the emergency of the COVID-19 pandemic, which has largely monopolised the attention of all the major EU institutions throughout most of the early months of the year, but also by the constant increase in morbidity, which now affects more than its ageing population. With the initiation of a European Health Data Space [54] and a new Pharmaceutical Strategy [55], 2020 is an appropriate moment also for it to review its approach to diagnostic testing as an intrinsic element in an integrated health strategy. In these straitened circumstances, a reappraisal of the significance and potential of biomarker testing in AD can provide the EU and its citizens with a much-needed immediate up-tick in quality and accuracy of care. The COVID-19 pandemic should also provide a warning of how vulnerable society is to inadequacies in healthcare preparation - and should serve to highlight the risk that dementia could, if unchecked, present a pandemic of similar or greater proportions within decades. Europe, with real foresight, can now make a change in policy that holds out the prospect of a radical transformation of care in the coming years as the full benefits of an optimum approach to the use of biomarkers begin to be felt.

\section{Recommendations}

\section{EU Recommendations}

- Declare early diagnosis and appropriate treatment of AD as a public health priority

- Promote standardisation in testing guidelines, validation, usage, quality control, and consistency of biomarkers

- Promote cross-sectoral collaboration and interdisciplinary research to advance the understanding of $\mathrm{AD}$

- Promote investment in basic and applied science to improve the understanding of AD

- Promote the integration of biomarker research into clinical medicine

- Promote bringing biomarker testing innovations to the market

- Promote coordination of clinical trials

- Promote alignment between innovators, payers, governments, businesses, and societies on what constitutes therapeutic and diagnostic value in $\mathrm{AD}$

\section{EMA Recommendations}

- Promote the use of biomarkers in regulatory decision making

- Increase the evidence base and resolve outstanding questions over interpretation of $\mathrm{AD}$ biomarker use and promote take-up

- Improve the understanding of biomarker testing among regulators

- Promote frequent interaction between industry and regulators

- Ensure globally accepted definitions of preclinical and early AD

- Promote the use of big data and the evolution of more flexible clinical trials

Member States Recommendations

- Promote the use of biomarkers in primary care and train general practitioners accordingly

- Ensure closer involvement of patients and caregivers in discussions of testing

- Improve understanding of biomarker testing among payers

- Ensure adequate infrastructure for diagnostic services

\section{Karger'}




\section{Healthcare Systems}

- All stakeholders to collaborate to enhance understanding of AD

- Patients, carers, and relevant associations to provide reliable information on the value of early detection and diagnosis of $\mathrm{AD}$

- Academia and industry to collaborate more closely on the development of biomarkers

- Improve business transparency over development costs of biomarker tests

- Develop less-invasive and cheaper and easier biomarkers

- Support the integration of AD biomarker testing within the AD diagnostic pathway

\section{Acknowledgements}

We would like to thank the members of the European Alliance for Personalised Medicine, the many experts for their work in this area, and the Million European Genome Alliance+ (MEGA+).

\section{Conflict of Interest Statement}

The authors declare that they have no competing interests.

\section{Funding Sources}

This work was supported by the in-kind support of the members of the European Alliance for Personalised Medicine.

\section{Author Contributions}

D.H. conceived, built, and drafted the article with critical input from his co-authors, expert interviews, and the partners.

\section{References}

1 European Commission. New scientific standards to help early diagnosis of Alzheimer's disease. Cited June 16, 2020. Available from: https://ec.europa.eu/luxembourg/news/new-scientific-standards-help-early-diagnosis-alzheimers-disease_fr

2 Hamermesh RG, Giusti K, Gulick S. Dementia Discovery Fund. Harvard Business School Case 820-045, September 2019.

3 Alzheimer Europe. The value of knowing - findings of Alzheimer Europe's five country survey on public perception of Alzheimer's disease and view on the value of diagnosis. Luxembourg: Alzheimer Europe; 2011.

4 Edith Cowan University. Newly identified biomarkers offers fresh hope for early Alzheimer's diagnosis, Medicalx Press; 2020 [cited 2020 Jun 16]. Available from: https://medicalxpress.com/news/2020-03-newlybiomarker-fresh-early-alzheimer.html.

5 NIH. Biomarkers for Dementia Detection and Research [cited 2020 Jun 16]. Available from: https://www.nia. nih.gov/health/biomarkers-dementia-detection-and-research.

6 NIH. Alzheimer's Disease Fact Sheet [cited 2020 Jun 16]. Available from: https://www.nia.nih.gov/health/ alzheimers-disease-fact-sheet.

7 Yiannopoulou KG, Papageorgiou SG. Current and future treatments for Alzheimer's disease. Ther Adv Neurol Disord. 2013 Jan;6(1):19-33.

8 Dubois B, Padovani A, Scheltens P, Rossi A, Dell'Agnello G. Timely diagnosis for Alzheimer's disease: a literature review on benefits and challenges. J Alzheimers Dis. 2016;49(3):617-31. 
9 Dubois B, Hampel H, Feldman HH, Scheltens P, Aisen P, Andrieu S, et al. Preclinical Alzheimer's disease: definition, natural history, and diagnostic criteria. Alzheimers Dement. 2016;12(3):292-323.

10 Albert MS, DeKosky ST, Dickson D, Dubois B, Feldman HH, Fox NC, et al. The diagnosis of mild cognitive impairment due to Alzheimer's disease: Recommendations for the National Institute on Aging-Alzheimer's Association workgroups on diagnostic guidelines for Alzheimer's disease. Alzheimers Dement. 2011;7(3):270-9.

11 McKhann GM, Knopman DS, Chertkow H, Hyman BT, Jack CR Jr, Kawas CH, et al. The diagnosis of dementia due to Alzheimer's disease: Recommendations from the National Institute of Aging-Alzheimer Association workgroups on diagnostic guidelines for Alzheimer's disease. Alzheimers Dement. 2011;7(3):263-9.

12 Jack CR Jr, Bennett DA, Blennow K, Carrillo MC, Dunn B, Haeberlein SB, et al. NIA-AA Research Framework: toward a biological definition of Alzheimer's disease. Alzheimers Dement. 2018;14(4):535-62.

13 Hwang AB, Boes S, Nyffeler T, Schuepfer G. Validity of screening instruments for the detection of dementia and mild cognitive impairment in hospital inpatients: A systematic review of diagnostic accuracy studies. PLoS One. 2019;14(7):e0219569.

14 Silverberg NB, Ryan LM, Carrillo MC, Sperling R, Petersen RC, Posner HB, et al. Assessment of cognition in early dementia. Alzheimers Dement. 2011;7(3):e60-76.

15 World Wide Fingers [Internet]. 2020 [cited 2020 June 16]. Available from: http://wwfingers.com.

16 Khan T. Introduction to Alzheimer's disease biomarkers. In: Biomarkers in Alzheimer's disease. Cambridge: Academic Press; 2016. pp. 3-23.

17 NIH. Neurological Diagnostic Tests and Procedures Fact Sheet [cited 2020 June 16]. Available from: https:// www.ninds.nih.gov/Disorders/Patient-Caregiver-Education/Fact-Sheets/Neurological-Diagnostic-Testsand-Procedures-Fact.

18 Khan TK. Neuroimaging biomarkers in Alzheimer's disease. In: Biomarkers in Alzheimer's disease. Cambridge: Academic Press; 2016. pp. 51-100.

19 Suppiah S, Didier MA, Vinjamuri S. The who, when, why, and how of PET amyloid imaging in management of Alzheimer's disease - review of literature and interesting images. Diagnostics. 2019;9(2):65.

20 Blennow K, Zetterberg H. Biomarkers for Alzheimer's disease: current status and prospects for the future. J Intern Med. 2018;284(6):643-63.

21 Alić I, Goh PA, Murray A, Portelius E, Gkanatsiou E, Gough G, et al. Patient-specific Alzheimer-like pathology in trisomy 21 cerebral organoids reveals BACE2 as a gene dose-sensitive AD suppressor in human brain [published online ahead of print, 2020 Jul 10]. Mol Psychiatry. 2020. https://doi.org/10.1038/s41380-0200806-5.

22 Karikari TK, Pascoal TA, Ashton NJ, Janelidze S, Benedet AL, Rodriguez JL, et al. Blood phosphorylated tau 181 as a biomarker for Alzheimer's disease: a diagnostic performance and prediction modelling study using data from four prospective cohorts. Lancet Neurol. 2020;19(5):422-33.

23 Janelidze S, Mattsson N, Palmqvist S, Smith R, Beach TG, Serrano GE, et al. Plasma P-tau181 in Alzheimer's disease: relationship to other biomarkers, differential diagnosis, neuropathology and longitudinal progression to Alzheimer's dementia. Nat Med. 2020;26(3):379-86.

24 Thijssen EH, La Joie R, Wolf A, Strom A, Wang P, Iaccarino L, et al. Diagnostic value of plasma phosphorylated tau181 in Alzheimer's disease and frontotemporal lobar degeneration. Nat Med. 2020;26(3):387-97.

25 Verberk IM, Slot RE, Verfaillie SC, Heijst H, Prins ND, van Berckel BN, et al. Plasma amyloid as prescreener for the earliest Alzheimer pathological changes. Ann Neurol. 2018;84(5):648-58.

26 Mitrečić D, Petrović DJ, Stančin P, Isaković J, Zavan B, Tricarico G, et al. How to face the aging world - lessons from dementia research. Croat Med J. 2020;61(2):139-46.

27 Swarbrick S, Wragg N, Ghosh S, Stolzing A. Systematic review of miRNA as biomarkers in Alzheimer's disease. Mol Neurobiol. 2019;56(9):6156-67.

28 Khan TK. Genetic biomarkers in Alzheimer's disease. In: Biomarkers in Alzheimer's Disease. Cambridge: Academic Press; 2016. pp. 103-35.

29 OECD. Global action to drive innovation in Alzheimer's disease and other dementias: connecting research, Regulation and access. OECD Science, Technology and Industry Policy Papers, No. 31. Paris: OECD Publishing; 2016.

30 de Wilde A, van Maurik IS, Kunneman M, et al. Alzheimer's biomarkers in daily practice (ABIDE) project: rationale and design. Alzheimers Dement. 2017;6:143-51.

31 van der Flier WM, Kunneman M, Bouwman FH, Petersen RC, Smets EMA. Diagnostic dilemmas in Alzheimer's disease: room for shared decision making. Alzheimers Dement. 2017;3(3):301-4.

32 Ferreira D, Perestelo-Pérez L, Westman E, Wahlund LO, Sarría A, Serrano-Aguilar P. Meta-review of CSF core biomarkers in Alzheimer's disease: the state-of-the-art after the new revised diagnostic criteria. Front Aging Neurosci. 2014;6:47.

33 Dyer SM, Flicker L, Laver K, Whitehead C, Cumming R. The clinical value of fluid biomarkers for dementia diagnosis. Lancet Neurol. 2016;15(12):1204.

34 Shaw LM, Arias J, Blennow K, Galasko D, Molinuevo JL, Salloway S, et al. Appropriate use criteria for lumbar puncture and cerebrospinal fluid testing in the diagnosis of Alzheimer's disease. Alzheimers Dement. 2018; 14(11):1505-21.

35 Nobili F, Arbizu J, Bouwman F, Drzezga A, Agosta F, Nestor P, et al. European Association of Nuclear Medicine and European Academy of Neurology recommendations for the use of brain 18 F-fluorodeoxyglucose positron emission tomography in neurodegenerative cognitive impairment and dementia: delphi consensus. Eur J Neurol. 2018;25(10):1201-17. 
36 Herukka SK, Simonsen AH, Andreasen N, Baldeiras I, Bjerke M, Blennow K, et al. Recommendations for cerebrospinal fluid Alzheimer's disease biomarkers in the diagnostic evaluation of mild cognitive impairment. Alzheimers Dement. 2017;13(3):285-95.

37 Blennow K, Zetterberg $\mathrm{H}$. The past and the future of Alzheimer's disease fluid biomarkers. J Alzheimers Dis. 2018;62(3):1125-40.

38 Lippa CF, Rosso AL, Stutzbach LD, Neumann M, Lee VM, Trojanowski JQ. Transactive response DNA-binding protein 43 burden in familial Alzheimer disease and Down syndrome. Arch Neurol. 2009;66(12):1483-8.

39 Khan TK. Alzheimer's disease cerebrospinal fluid (CSF) biomarkers. In: Biomarkers in Alzheimer's disease. Cambridge: Academic Press; 2016. pp. 139-80.

40 Altomare D, Ferrari C, Caroli A, Galluzzi S, Prestia A, van der Flier WM, et al. Prognostic value of Alzheimer's biomarkers in mild cognitive impairment: the effect of age at onset. J Neurol. 2019;266(10):2535-45.

41 Lee JC, Kim SJ, Hong S, Kim Y. Diagnosis of Alzheimer's disease utilizing amyloid and tau as fluid biomarkers. Exp Mol Med. 2019;51(5):1-10.

42 Hlavka JP, Mattke S, Liu JL. Assessing the preparedness of the health care system infrastructure in six European countries for an Alzheimer's treatment. Rand Health Q. 2019;8(3):2.

43 Eurostat. Physicians by Medical Speciality. 2017 [cited 2020 June 16]. Avaibale from: http://appsso.eurostat. ec.europa.eu/nui/show.do?dataset=hlth_rs_spec\&lang=en.

44 Royal College of Psychiatrists. Census 2017: Workforce figures for consultant and specialty doctor psychiatrists. 2017 [cited 2020 June 16]. Available from: https://www.rcpsych.ac.uk/pdf/RCPsych_workforce_ census_report_2017.pdf.

45 Bradley P, Akehurst R, Ballard C, Banerjee S, Blennow K, Bremner J, et al. Taking stock: A multistakeholder perspective on improving the delivery of care and the development of treatments for Alzheimer's disease. Alzheimers Dement. 2015;11(4):455-61.

46 Conejero I, Navucet S, Keller J, Olié E, Courtet P, Gabelle A. A complex relationship between suicide, dementia and amyloid: a narrative review. Front Neurosci. 2018;12:371.

47 Hort J, O'Brien JT, Gainotti G, Pirttila T, Popescu BO, Rektorova I, Sorbi S, Scheltens P, EFNS Scientist Panel on Dementia. EFNS guidelines for the diagnosis and management of Alzheimer's disease. Eur J Neurol. 2010;17: 1236-48.

48 Watson R, et al. What is a 'timely' diagnosis? Exploring the preferences of Australian health service consumers regarding when a diagnosis of dementia should be disclosed. BMC Health Serv Res. 2018:18(1):612.

49 Alzheimer's Association. 2020 Alzheimer's disease facts and figures. Alzheimers Dement. 2020;16(3):391460.

50 Wimo A, Ballard C, Brayne C, Gauthier S, Handels R, Jones RW, et al. Health economic evaluation of treatments for Alzheimer's disease: impact of new diagnostic criteria. J Intern Med. 2014;275(3):304-16.

51 Innovative Medicines Initiative. EPAD - revolutionising clinical trials for dementia. 2020 [cited 2020 Jun 16]. Available from: https://www.imi.europa.eu/projects-results/success-stories-projects/epad-revolutionising-clinical-trials-dementia.

52 Us Against Alzheimer's, Global Alzheimer's Platform and Innovative Medicines Initiative to Sign Memorandum of Understanding to Accelerate Alzheimer's Drug Development [cited 2020 Jun 16]. Available from: https:// www.usagainstalzheimers.org/press/global-alzheimer's-platform-and-innovative-medicines-initiativesign-memorandum-understanding.

53 NIH. New biological research framework for Alzheimer's seeks to spur discovery [cited 2020 Jun 16]. Available from: https://www.nia.nih.gov/news/new-biological-research-framework-alzheimers-seeks-spur-discovery.

54 European Commission. Communication from the Commission to the European Parliament, the Council, the European Economic and Social Committee and the Committee of the Regions, on enabling the digital transformation of health and care in the Digital Single Market; empowering citizens and building a healthier society. COM(2018) 233 final. Brussels, 2018 [cited 2020 Jun 16]. Available from: https://eur-lex.europa.eu/legalcontent/EN/TXT/HTML/?uri=CELEX:52018DC0233\&from=EN.

55 European Commission. Pharmaceutical strategy for Europe [cited 2020 Jun 16]. Available from: https:// ec.europa.eu/health/human-use/strategy_en.

56 Alzforum. Deadline to weigh in on new biomarker guidance approaches [Internet]. 2019. Available from: https://www.alzforum.org/news/community-news/deadline-weigh-new-biomarker-guidance-approaches.

57 Gotovac K, Pivac N, Hajnšek S, et al. Quest for new genomic and proteomic biomarkers in neurology. Translat Neurosci. 2011;2:69-75.

58 Grand View Research [Internet]. Neurological biomarkers market size, industry trend report, 2019-2026. San Francisco: GVR; 2019. Available from: www.grandviewresearch.com.

59 Arnerić SP, Kern VD, Stephenson DT. Regulatory-accepted drug development tools are needed to accelerate innovative CNS disease treatments. Biochem Pharmacol. 2018;151:291-306. 